\title{
Certificación energética de un edificio de oficinas
}

Energy certification of an office building

\section{Miguel Castro-Fernández¹, Miriam Vilaragut Llanes², Aziz Oloroun-Shola Bissiriou ${ }^{3}$}

Castro-Fernández, M; Vilaragut-Llanes, M; Oloroun-Shola Bissiriou, A. Certificación energética de un edificio de oficinas. Tecnología en Marcha. Vol. 34, especial. ALTAE. Diciembre 2021. Pág 70-82.

doi) https://doi.org/10.18845/tm.v34i7.6015

1 CIPEL, Cuba. Correo electrónico: mcastro@electrica.cujae.edu.cu (D) https://orcid.org/0000-0002-3983-469X

2 CIPEL, Cuba. Correo electrónico: miriamv@electrica.cujae.edu.cu (D) https://orcid.org/0000-0002-5453-1136

3 Centro de Investigaciones y Pruebas Electroenergéticas (CIPEL), Cuba 


\title{
Palabras clave
}

Certificación energética, ahorro de energía, disminución de gases efecto invernadero.

\section{Resumen}

La certificación energética se presenta como una herramienta de gran utilidad al ofrecer información sobre la eficiencia energética, los consumos energéticos asociados y las emisiones de $\mathrm{CO} 2$ que dichos consumos generan, por lo que buscar oportunidades de ahorro de energía eléctrica en edificaciones mediante la certificación energética, se presenta como una oportunidad que en Cuba no ha sido explotada aún. En este trabajo se desarrolla un estudio sobre la aplicación del procedimiento que se aplica en la Comunidad Europea para certificar las edificaciones ya construidas, y se proponen un grupo de medidas de eficiencia energética y el posible uso de Fuentes Renovables de Energía en el edificio bajo estudio, con el objetivo de elevar la certificación inicial otorgada. Para ello se aplica el software CE3X, el cual permite evaluar los indicadores de consumo energético y la cantidad de emisiones de $\mathrm{CO}_{2}$, asociadas a dicho consumo, que se libera a la atmosfera. Como conclusión se obtiene que el edificio presenta un alto consumo de energía, siendo un elemento fundamental el consumo que tiene en el sistema de climatización. Este problema permitió plantear propuestas detalladas obteniendo como resultado un ahorro de energía anual del $43 \%$ y del $52 \%$ de las emisiones globales de $\mathrm{CO}_{2}$. Las medidas propuestas para lograr los anteriores se justifican económicamente por los valores del Valor Actual Neto (VAN) calculado, a partir de una inversión total para satisfacer las mejoras escogidas de 36 300,00 €, estimándose que en 8 años toda la inversión habrá sido recuperada.

\section{Keywords}

Energy certification, energy saving, greenhouse gas reduction.

\begin{abstract}
Energy certification is presented as a very useful tool by offering information on energy efficiency, associated energy consumption and the $\mathrm{CO} 2$ emissions that such consumption generates, so looking for opportunities to save electricity in buildings through energy certification, It is presented as an opportunity that in Cuba has not yet been exploited. In this work, a study is developed on the application of the procedure applied in the European Community to certify buildings already built, and a group of energy efficiency measures and the possible use of Renewable Energy Sources in the building underneath are proposed. study, with the objective of raising the initial certification granted. For this, the CE3X software is applied, which allows evaluating the energy consumption indicators and the amount of $\mathrm{CO} 2$ emissions, associated with said consumption, which is released into the atmosphere. As a conclusion, it is obtained that the building has a high energy consumption, being a fundamental element the consumption that it has in the air conditioning system. This problem allowed for detailed proposals, resulting in $43 \%$ annual energy savings and $52 \%$ of global CO2 emissions. The measures proposed to achieve the above are economically justified by the values of the Net Present Value (NPV) calculated, based on a total investment to satisfy the chosen improvements of $€ 36,300.00$, estimating that in 8 years the entire investment will have been recovered.
\end{abstract}




\section{Introducción}

La eficiencia energética de un edificio se determina calculando o midiendo el consumo de energía necesaria para satisfacer anualmente la demanda energética del edificio en unas condiciones normales de funcionamiento y ocupación, y se expresa de forma cualitativa o cuantitativa mediante indicadores, y calificación, o letras de una escala determinada convencionalmente y que varía de mayor a menor eficiencia, tal y como se muestra en la figura 1.

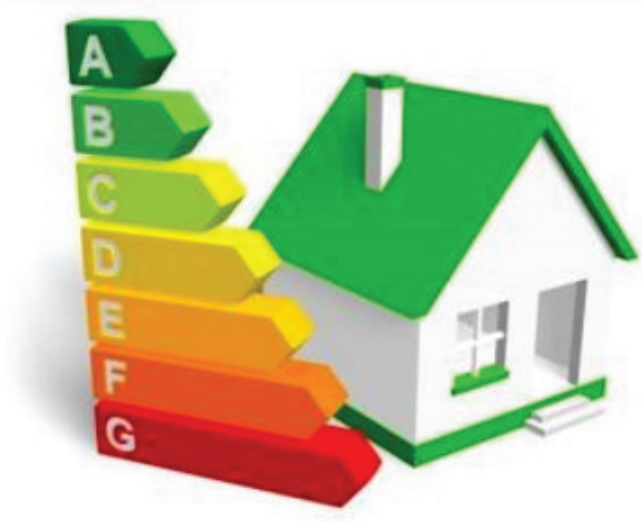

Figura 1. Etiquetado de una edificación. Fuente: El Certificado Obligatorio de Eficiencia Energética cumple su primer año (2014).

En el presente trabajo se realiza la certificación energética de un edificio de oficinas con el fin de establecer un punto de partida, sobre la base de experiencias internacionales, y buscar oportunidades de ahorro de energía eléctrica en las edificaciones, no sólo por las características de los equipos que se utilizan sino también de la propia edificación. Para lograr lo anterior se plantean diferentes medidas para intentar reducir tanto el gasto de las facturas de electricidad, como las emisiones del edificio y se estudian la viabilidad de estas mejoras en todos los aspectos. También se realiza un análisis de la viabilidad donde se tuvieron en cuenta los factores energéticos, económicos y otro tipo de aspectos ajenos, como permisos externos necesarios.

Por tal razón, los autores se plantearon como problema a solucionar las siguientes interrogantes: ¿es posible mejorar la eficiencia energética del edificio? ¿Se podrán obtener resultados favorables en la gestión energética de la empresa, a partir de aplicar las mejoras propuestas? Para dar respuesta a estas interrogantes se decide aplicar el software CE3X, como una posible herramienta para la evaluación energética inicial y el análisis posterior de los posibles resultados si se aplican las medidas propuestas, llevando a una mejora de la eficiencia energética del edificio.

Si se define como eficiencia energética a la proporción o relación cuantitativa entre el resultado en términos de desempeño, de servicios, de bienes o de energía y la entrada de energía, se observa la necesidad, para el sector de la industria y/o servicios, de definir indicadores de desempeño energético (IDEs) característicos para cada uno de ellos, e incluso a nivel de procesos, y para que estos indicadores alcancen los valores deseados, la eficiencia energética debe entonces, por demás, ser gestionada para alcanzar su mayor potencial ayudando también a reducir emisiones y a disminuir la intensidad energética.

En el caso del sector inmobiliario, donde muchas veces radican entidades que son responsables de producciones y/o servicios a terceros, se debía también buscar indicadores que permitieran definir la eficiencia energética de estas instalaciones; es por eso que, con el objeto de promover la eficiencia energética de los edificios, la Unión Europea estableció la Directiva 2002/91/CE la 
cual exigía, a los Estados miembros, el establecimiento de un procedimiento de certificación, de manera tal que pusiese a disposición del posible comprador o inquilino una información objetiva sobre el consumo energético de la edificación y/o del apartamento en específico. La eficiencia energética de un edificio se calcula midiendo la energía que se consume durante un año en condiciones normales de uso y ocupación en cuanto a calefacción, agua caliente sanitaria, ventilación, iluminación. Estos datos expresan valores finales de consumo de energía, medidos en kilowatts hora por metro cuadrado $\left(\mathrm{kWh} / \mathrm{m}^{2}\right.$.año) y en kilogramos de $\mathrm{CO}_{2}$ por metro cuadrado de vivienda ( $\mathrm{kg} \mathrm{CO} / \mathrm{m}^{2}$. año) que se corresponden con una letra de la escala de eficiencia energética en edificios, o lo que es lo mismo: la eficiencia energética de un edificio se establece a partir de definir una escala de uso de los indicadores de emisiones anuales de $\mathrm{CO}_{2}$ y el consumo anual de energía primaria no renovable que registra una edificación o vivienda en particular. Esta escala se muestra en la figura 2.
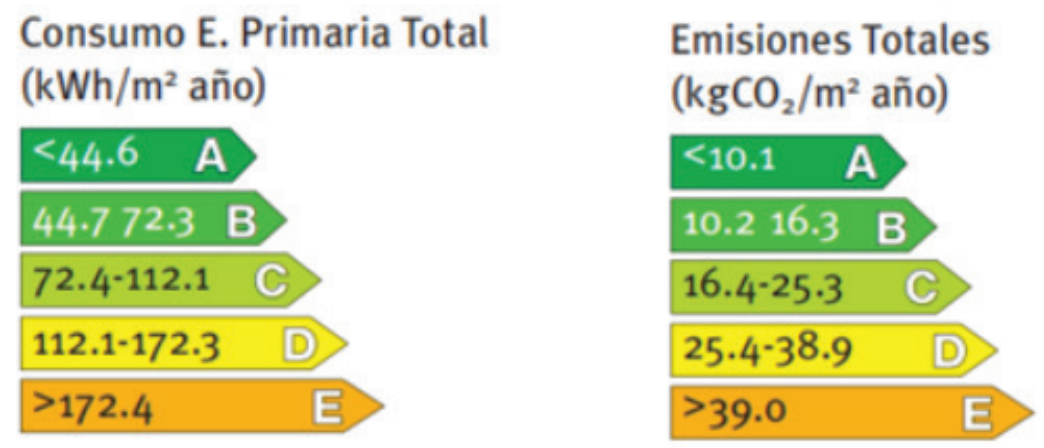

Figura 2. Escala de calificación energética para los edificios según los indicadores expuestos.

Fuente: (Instituto para la Diversificación y Ahorro de la Energía (IDAE) 2009).

A partir de esta clasificación una edificación que obtenga la categoría A puede consumir hasta un $90 \%$ menos de energía que una que esté catalogada con el nivel más bajo, y lograr esta condición va acompañada de la aplicación de un grupo de medidas que consiguen reducir el consumo energético de la instalación.

\section{Caracterización del edificio bajo estudio}

El edificio bajo estudio cuenta con 4 niveles de plantas de oficinas y está orientado al noroeste.

El paso del tiempo y la falta de mantenimiento del edificio han dejado su huella en cuanto a las condiciones actuales del edificio, aun cuando se ha sometido a varias reparaciones interiores y mejoras en cuanto a equipamiento utilizado.

En un informe preliminar realizado por los autores, a partir de los datos aportados por la empresa que ocupa el edificio referente a sus consumos históricos y el uso de los portadores energéticos, se llegó a la conclusión que el mismo podía ser calificado, preliminarmente, con la letra $\mathrm{D}$, ya que presentaba niveles de consumo anual de energía primaria no renovable, dado en $\mathrm{kWh} / \mathrm{m}^{2}$. año de 116,7 y de emisiones anuales de $\mathrm{CO}_{2}$ del orden de los $26,84 \mathrm{~kg}$ de $\mathrm{CO}_{2} / \mathrm{m}^{2}$. año (Castro et al, 2019). La situación problemática que se presentaba era, entonces, disminuir los niveles de consumo de energía primaria no renovable y de las emisiones al ambiente de $\mathrm{CO}_{2}$ a niveles aceptables, introduciendo medidas y equipamiento más eficiente en la instalación y en las actividades que allí se realizan. 
El sistema de distribución de energía eléctrica que existe en la instalación bajo estudio se resume de la forma siguiente: se alimenta de un circuito de alta tensión a través de un transformador de $13 \mathrm{kV}$ a 208/120 V en estrella (Y) con neutro a tierra. Este transformador alimenta un interruptor de 800 A regulado a 400 A que alimenta la PGD de la instalación compuesta por un panel donde están ubicados un conjunto de interruptores encargados de la distribución eléctrica del edificio. Así mismo, de acuerdo a los datos aportados por la empresa que ocupa el edificio el 99,9\% del consumo de portadores energéticos es responsabilidad del consumo de electricidad, siendo la estructura del consumo de las cargas instaladas en el edificio la que se muestra en la Figura 3.

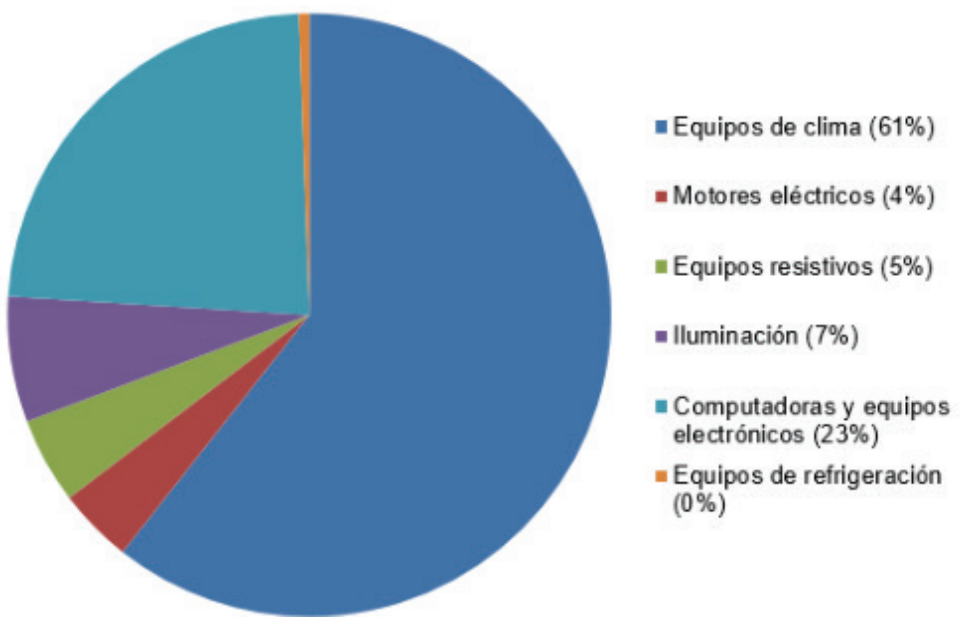

Figura 3. Participación de las cargas instaladas en el consumo de electricidad del edificio bajo estudio.

El sistema de climatización del edificio bajo estudio ha tenido muy pocos cambios desde su instalación original, y actualmente es muy poco eficiente, existiendo muchas pérdidas tanto en verano como en invierno. La primera opción para un buen rendimiento térmico, en las condiciones de Cuba, es reducir las ganancias de calor en verano, disminuyendo de este modo la demanda de energía necesaria para el acondicionamiento térmico del edificio. Estas transferencias de calor van a depender en primer lugar de las características constructivas del edificio. Desde el punto de vista climático, se considera que las oficinas y salas de reuniones que se encuentran orientadas hacia la fachada suroeste están mal ubicadas por ser localizadas hacia la orientación menos favorable en cuanto a la dirección de las brisas predominantes y asoleamiento, desaprovechando las brisas predominantes que vienen desde la orientación noreste en el caso cubano. Así mismo, esa fachada se encontrará muy afectada por los rayos incidentes del sol provocando el calentamiento de las superficies y el aire interior, afectando el confort térmico. Esta última requerirá de un nivel muy alto de energía para garantizar las condiciones de confort a partir del empleo de sistemas de climatización ubicados en las oficinas de dicha fachada.

Como se puede observar de la figura 3, los mayores gastos (61\%) de la energía consumida en el edificio tiene como elemento principal la climatización del mismo, siendo el gasto correspondiente a iluminación y refrigeración mínimo con respecto a las que corresponden al clima. A todo ello se debe agregar que:

- La instalación de la mayoría de los equipos de refrigeración data entre los años 1994 y 2013, con más de 10 años de explotación, por lo que dada sus antigüedades sus vidas útiles están casi terminadas. 
- El sistema de iluminación, fundamentalmente, está conformado por luminarias tipo LED, con una potencia total de instalación de 12,33 kW y una Densidad de Potencia Eléctrica para Alumbrado (DPEA) de $8,50 \mathrm{~W} / \mathrm{m}^{2}$, valor que está dentro de lo normado para las oficinas, tipo de local que forma parte, fundamentalmente, de este edificio.

- Por otro lado, la mayor parte de los aires acondicionados son muy viejos: unos no tienen chapa siquiera que especifique sus datos técnicos, mientras que en otros ni se ve lo que dice la chapa. Además, algunos que tienen chapa y después de tanto tiempo puede que se hayan reparado y lo que dice la chapa no sea real.

Por lo anterior expuesto, en este trabajo se utilizaron datos estimados para el cálculo de los equipos de aire acondicionado con un rendimiento nominal del $150 \%$. Basta recordar que mientras más alta sea el porciento del rendimiento nominal más eficiente será el equipo de clima.

Así mismo, para realizar la certificación energética de un edificio se requiere también conocer un pequeño grupo de datos generales, los cuales se presentan en el cuadro 1.

Cuadro 1. Datos generales.

\begin{tabular}{|c|c|}
\hline Región o provincia: & La Habana \\
\hline Zona climática: & A3 ${ }^{4}$ \\
\hline Año de construcción: & 1930 \\
\hline Normativa vigente de construcción: & Anterior a 1930 \\
\hline Superficie útil (en metros cuadrados): & 3615 \\
\hline Altura libre de planta (en metros): & $14,62^{5}$ \\
\hline Número de plantas utilizables: & 4 \\
\hline Ventilación del inmueble: & 0,8 \\
\hline Demanda diaria de ACS (en metros cúbicos): & 0 \\
\hline
\end{tabular}

Por otra parte, se requiere conocer las características de la envolvente térmica del edificio. La envolvente térmica se divide principalmente en muros de fachadas, huecos y lucernarios; posteriormente, los cerramientos y particiones interiores de los espacios habitables se clasificarán según su situación como cubiertas y muros. En el caso del edificio bajo estudio, la cubierta comprenderá aquellos cerramientos superiores en contacto con el aire y cuya inclinación sea inferior a $60^{\circ}$ respecto a la horizontal. El edificio bajo estudio no posee cubiertas que están en contacto con el terreno, sino una cubierta al aire y las otras son intermedias estando en contacto con otro piso. La cubierta exterior está compuesta de hormigón armado y aceros. En el caso de los muros, el edificio cuenta con cuatro fachadas siendo la orientación de cada fachada la que se muestra en la figura 4.

4 Según Darpón Marqués (2019)

5 Se calcula en función de la altura de cada habitación por la superficie de dicha habitación dividido entre la superficie de la habitación 


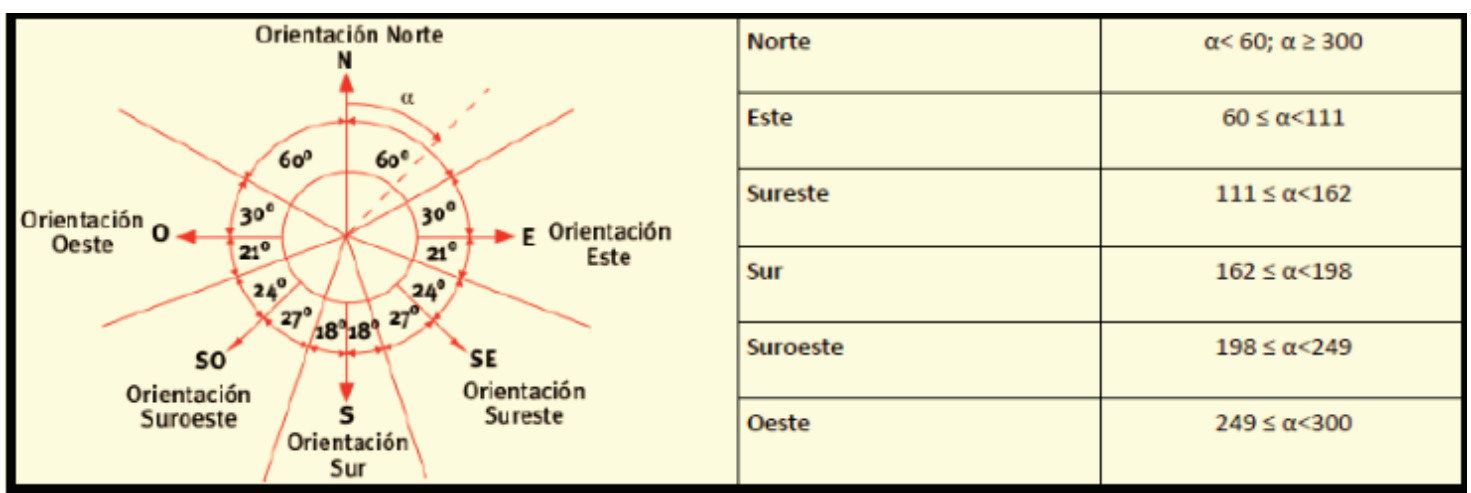

Figura 4. Orientación de fachadas. Fuente: Código Técnico de la Edificación de España (2006).

Por otro lado, los muros son de ladrillo y no presentan buen aislamiento térmico, pero hay que tener en cuenta que el edificio es muy antiguo. Del exterior al interior son los revoques, luego se tiene una capa de ladrillo y finalmente otra capa de revoque, siendo esta última de un espesor menor que la primera capa.

Las ventanas del edificio son de dos tipos: unas son de vidrio corredera y otras de persiana metálica. Las dimensiones de estas varían, pero todas tienen las mismas prestaciones.

Otro elemento importante, desde el punto de vista de la estructura del edifico es lo relacionado con los puentes térmicos. Esta es una zona puntual o lineal, de la envolvente de un edificio, en la que se transmite más fácilmente el calor que en las zonas aledañas, debido a una variación de la resistencia térmica. Se trata de un lugar en el que se rompe la superficie aislante. En el caso bajo estudio, el edificio muestra dos puentes térmicos característicos: pilar integrado en fachada y encuentro de fachada con cubierta.

\section{Evaluación desde el punto de vista de la eficiencia energética del edificio bajo estudio}

\section{Breve caracterización del software CE3X}

El software CE3X fue desarrollado por el Instituto para la Diversificación y Ahorro de la Energía (IDAE). Este programa forma parte del procedimiento reconocido para la certificación energética de edificios existentes en España, el cual a su vez ha sido desarrollado conjuntamente por el Centro Nacional de Energías Renovables (CENER) y el equipo de trabajo que desarrolló el CE3X. El software tiene una amplia base de datos de materiales, soluciones constructivas y propiedades térmicas en función de la antigüedad del edificio. Mediante este programa se puede certificar de una forma simplificada cualquier tipo de edificio y pudiéndose obtener cualquier calificación desde "A" hasta "G" tal y como se ha mostrado anteriormente (Escala de Calificación Energética, 2009). Basta recordar que la diferencia entre estos tres tipos de edificio está basada en las instalaciones que los componen. El software incorpora además una serie de conjuntos de medidas de mejora de eficiencia energética, la nueva calificación que la aplicación de cada conjunto de medidas de mejora supondría y la posibilidad de realizar un análisis económico del impacto de dichas medidas basado en los ahorros energéticos estimados por la herramienta o las facturas de consumo de energía.

El programa CE3X, a partir de los datos geométricos, localidad y características constructivas, bien sean definidas o por defecto, simula un año completo y calcula la demanda del edificio. Con los datos de la instalación, el programa calcula el consumo y a partir de ahí las emisiones. El proceso a seguir es el siguiente: 
- Se introducen los datos generales del edificio.

- Se analiza la envolvente térmica, por lo que habrá que definir todos los muros y sus propiedades, su posición y los datos de todos los huecos y lucernarios. Para ello se debe calcular previamente la transmitancia térmica de cada uno de los huecos, o calcularlos por defecto en función del material del que estén hechos.

- Una vez definida la envolvente térmica se introducen los datos de las instalaciones de las que disponga el edificio y las características de dichas instalaciones. Las instalaciones térmicas de un edificio son los equipos destinados a producción de ACS que no es más que el sistema de calefacción, refrigeración y tratamiento de aguas.

- Por último, el programa dará una calificación y ofrecerá diferentes mejoras, de las cuales se elegirán las adecuadas.

\section{Introducción de datos}

La entrada de datos en CE3X empieza con la introducción de los datos administrativos (ver figura 5), los datos generales y de definición del edificio (ver figura 6) y los datos de la envolvente térmica (ver figura 7). Para esta última información, se dividió el edificio en las cuatro plantas que lo conforman:

- La planta 1 corresponde al nivel 1, con una superficie útil de $1014 \mathrm{~m}^{2}$.

- La planta 2 corresponde al nivel 2, con una superficie útil es $960 \mathrm{~m}^{2}$.

- La planta 3 corresponde al nivel 3, con una superficie útil de $959 \mathrm{~m}^{2}$.

- La planta 4 corresponde al nivel 3, con una superficie de $624 \mathrm{~m}^{2}$.

\begin{tabular}{|c|c|c|c|c|c|c|}
\hline Nombre del edificio & \multicolumn{6}{|c|}{ EDIFICIO DEL CEDAI } \\
\hline Dirección & \multicolumn{6}{|c|}{ CALLE G ESQUINA 13 EN VEDADO } \\
\hline Provincia/Ciudad autónoma & Las Palmas & $\checkmark$ & Localidad & Las Palmas de Gran Canaria $\vee$ & Código Postal & 302 \\
\hline Referencia Catastral & $\mathrm{x}$ & & & & & \\
\hline \multicolumn{7}{|l|}{ Datos del cliente } \\
\hline Nombre o razón social & \multicolumn{6}{|c|}{ EDIFICIO DEL CEDAI } \\
\hline Dirección & \multicolumn{6}{|c|}{ CALLE G ESQUINA 13 EN VEDADO } \\
\hline Provincia/Ciudad autónoma & Las Palmas & $\checkmark$ & \multirow{2}{*}{$\begin{array}{l}\text { Localidad } \\
\text { E-mail }\end{array}$} & Otro & \multirow[t]{2}{*}{ Código Postal } & 302 \\
\hline Teléfono & & & & $\mathrm{x}$ & & \\
\hline \multicolumn{7}{|c|}{ Datos del técnico certificador } \\
\hline Nombre y Apellidos & \multicolumn{4}{|c|}{ AZIZ OLOROUN-SHOLA BISSIRIOU } & \multirow[t]{2}{*}{ NIF } & $\mathrm{x}$ \\
\hline Razón social & \multicolumn{4}{|l|}{ CIPEL } & & $\mathrm{x}$ \\
\hline Dirección & \multicolumn{6}{|c|}{ CALLE 114 CICLOVIA Y ROTONDA } \\
\hline Provincia/Ciudad autónoma & Las Palmas & $\checkmark$ & \multirow{2}{*}{$\begin{array}{l}\text { Localidad } \\
\text { E-mail }\end{array}$} & Otro & \multirow[t]{2}{*}{ Código Postal } & $\mathrm{x}$ \\
\hline Teléfono & $\mathrm{x}$ & & & $\mathrm{x}$ & & \\
\hline $\begin{array}{l}\text { Titulación habilitante según } \\
\text { normativa vigente }\end{array}$ & \multicolumn{6}{|c|}{ TRABAJO DE DIPLOMA PARA OPTAR POR EL TITULO DE INGENIERO ELECTRICISTA| } \\
\hline
\end{tabular}

Figura 5. Pantalla de introducción de datos administrativos. Fuente: foto de pantalla del software CE3X. 


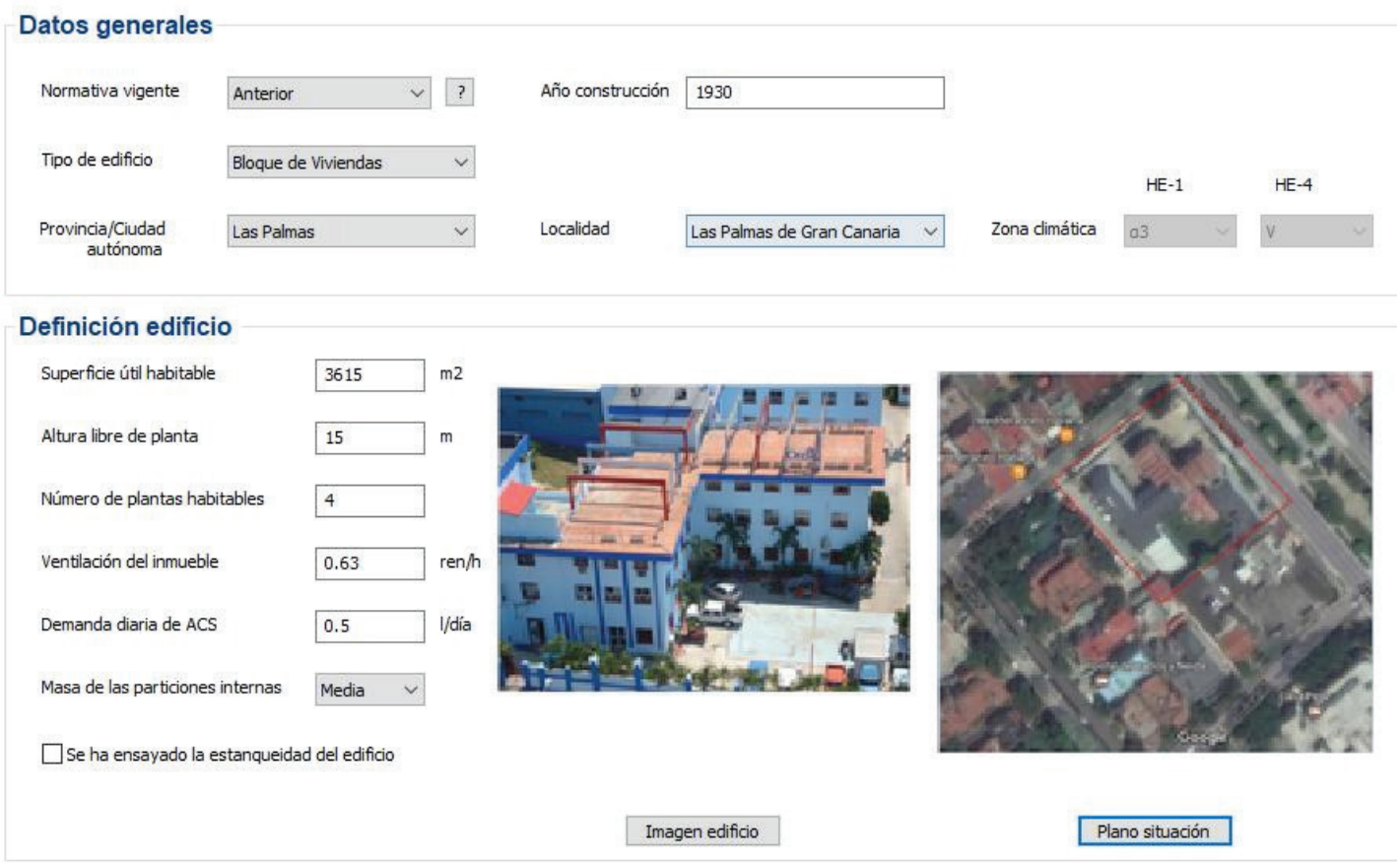

Figura 6. Pantalla de introducción de datos generales y definición del

edificio. Fuente: foto de pantalla del software CE3X.

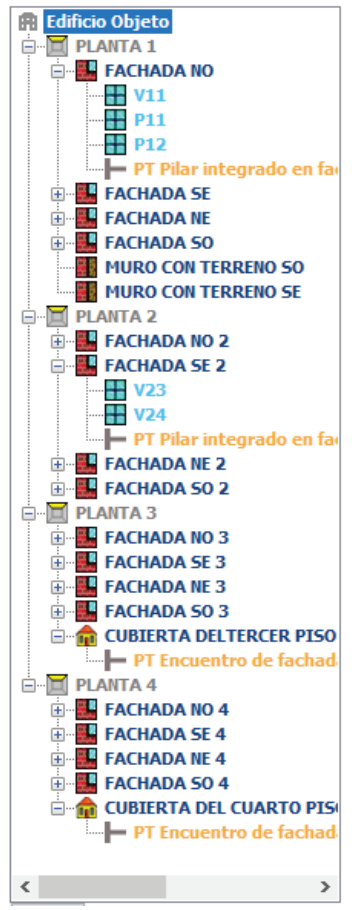

Envolvente térmica del edificio

Ocubierta

OMuro

Osuelo

Partición interior

Hueco/Lucernario

Puente térmico Definidos por usuario

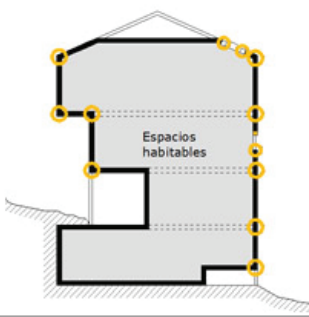

Puente térmico por defecto

Definir puentes térmicos por defecto

$\square$ Pilar integrado en fachada

$\square$ Pilar en esquina

$\square$ Contorno de hueco

$\square$ Caja de persiana

$\square$ Encuentro de fachada con forjado

$\square$ Encuentro de fachada con cubierta

$\square$ Encuentro de fachada con suelo en contacto con el aire

$\square$ Encuentro de fachada con solera

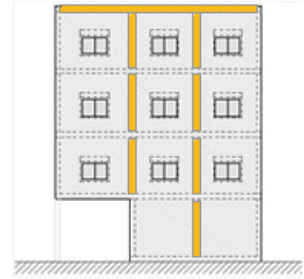

Figura 7. Pantalla de introducción de los datos de la envolvente térmica. Fuente: foto de pantalla del software CE3X. 
Por último, se entregan al software los datos referentes a la instalación de climatización, en la cual se supuso un porcentaje de demanda que es cubierta por la climatización en cada planta por los problemas expuestos anteriormente. La figura 8 muestra la pantalla donde se definen los parámetros para el cálculo, mientras que en el cuadro 2 se muestran los porcentajes de cubrimiento de las necesidades de climatización para cada planta.

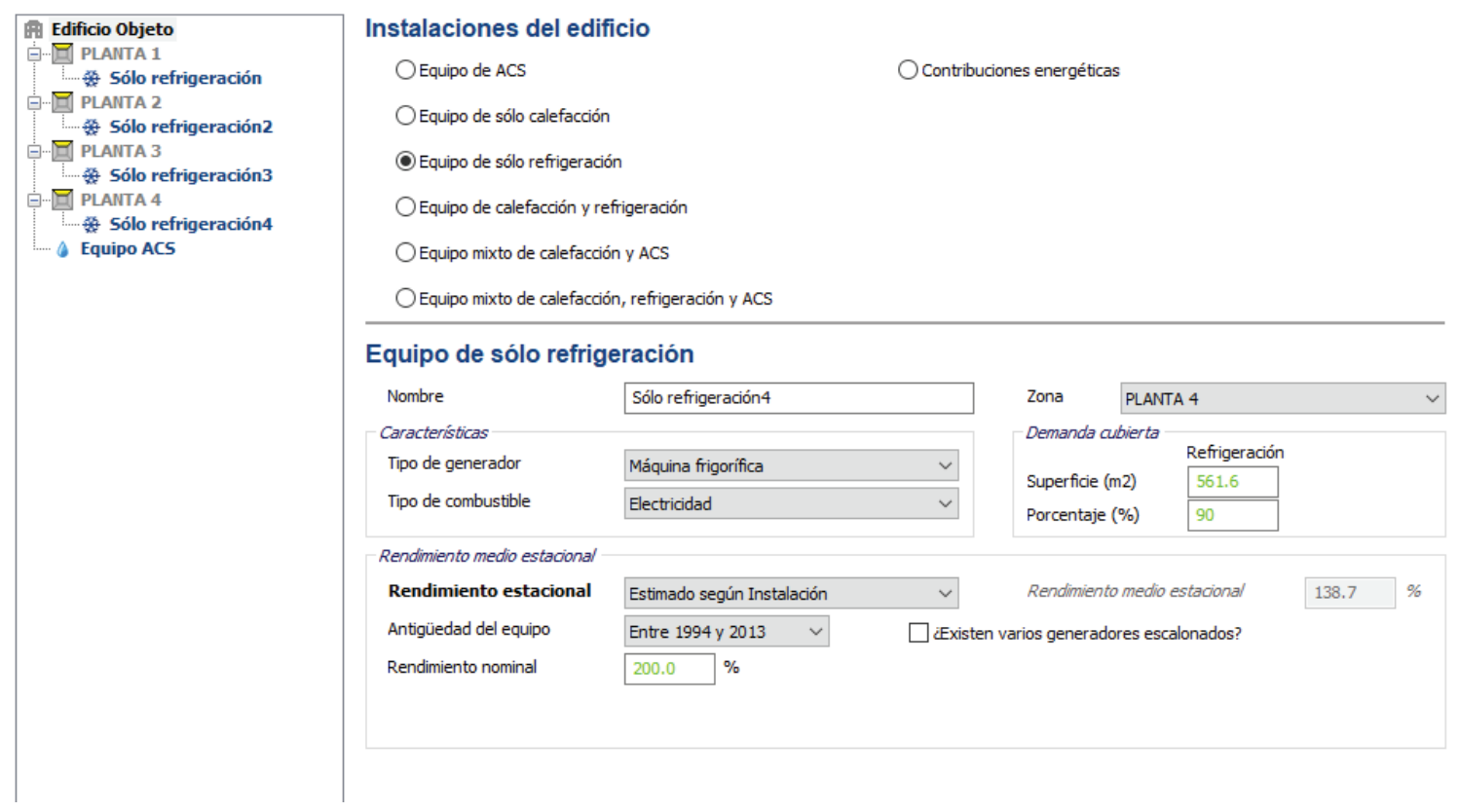

Figura 8. Pantalla de introducción de los datos de las instalaciones.

Fuente: Fuente: foto de pantalla del software CE3X.

Cuadro 2. Porcentaje de la demanda cubierta de la refrigeración.

\begin{tabular}{|c|c|c|c|}
\hline \multirow{2}{*}{ Plantas } & \multicolumn{3}{|c|}{ Demanda cubierta } \\
\cline { 2 - 4 } & Superficie útil $\left(\mathrm{m}^{2}\right)$ & Porcentaje $(\%)$ & Superficie $\left(\mathrm{m}^{2}\right)$ \\
\hline Planta 1 & 1014 & 80 & 811,2 \\
\hline Planta 2 & 960 & 85 & 816,0 \\
\hline Planta 3 & 959 & 90 & 863,1 \\
\hline Planta 4 & 624 & 92 & 574,08 \\
\hline
\end{tabular}

\section{Análisis de Resultados}

Tal y como se mencionó anteriormente, la evaluación preliminar desde el punto de vista de la energía ofreció una categorización de nivel D con 116,7 kWh/m². año consumidos en el edificio, mientras que en lo referente a emisiones anuales de $\mathrm{CO}_{2}$ los resultados indicaban una letra $\mathrm{G}$ con $26,84 \mathrm{~kg}$ de $\mathrm{CO}_{2} / \mathrm{m}^{2}$. año. Al aplicar el software, los resultados de consumo de energía se mantuvieron, pero en el caso de las emisiones, al incorporar conceptos relativos a la estructura del edificio y otros elementos los resultados alcanzados fueron los que se muestran en la figura 9 . 


\section{Calificación energética de edificios Indicador $\mathrm{kgC02} / \mathrm{m} 2$}

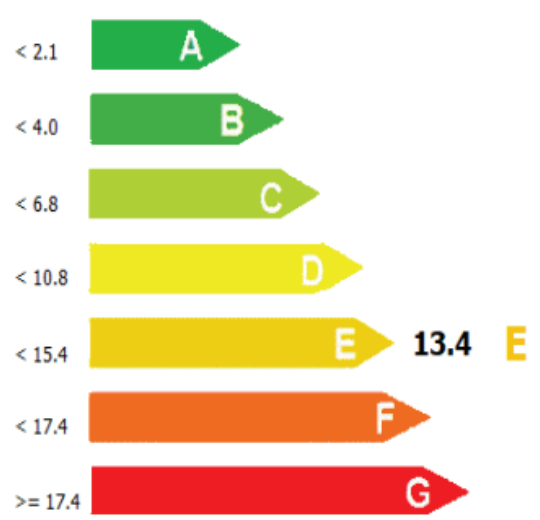

\section{Edificio objeto}

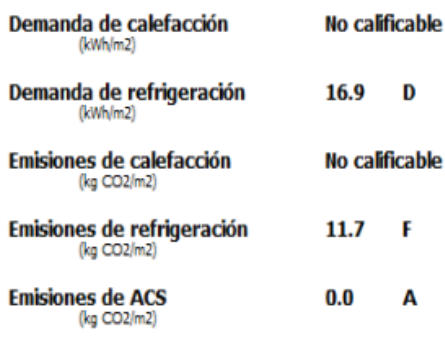

Figura 9. Calificación actual del edificio bajo estudio desde el punto de vista de las emisiones.

Fuente: foto de pantalla del software CE3X referente a la certificación energética final.

Partiendo de estos resultados, se propusieron un grupo de medidas para buscar la mejora de las certificaciones otorgadas, entre las cuales sobresalen:

1. Cambio de ventanas

2. Adición de aislamiento térmico de en las fachadas (suroeste) por el interior con el fin de reducir la demanda

3. Mejora de los huecos de las fachadas noroeste, sureste, suroeste y noreste.

4. Incorporación de energía solar para suplir parte de la alimentación que requiere el sistema de climatización.

La viabilidad de aplicación de estas medidas puede verse en las figuras 10 y 11, donde se analizan los paquetes desde el punto de vista de la estructura del edificio y la inclusión del sistema fotovoltaico.
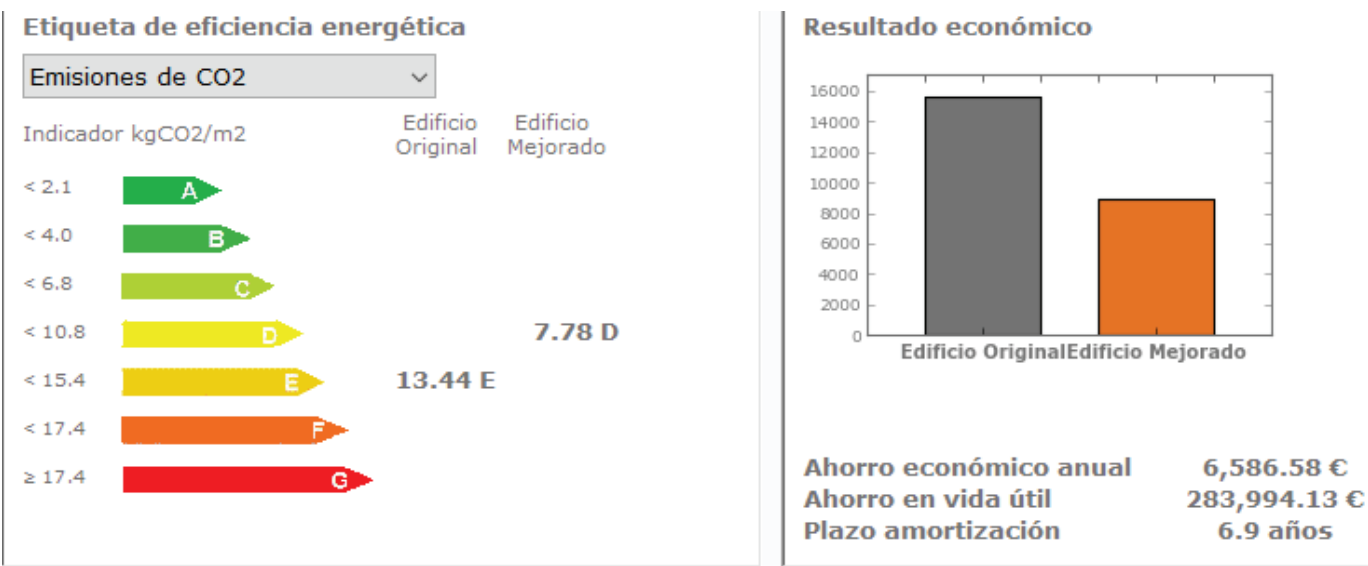

Figura 10. Viabilidad económica del conjunto de medidas propuestas desde el punto de vista estructural del edificio. Fuente: foto de pantalla del complemento Saltoki Instalaciones de CE3X. 

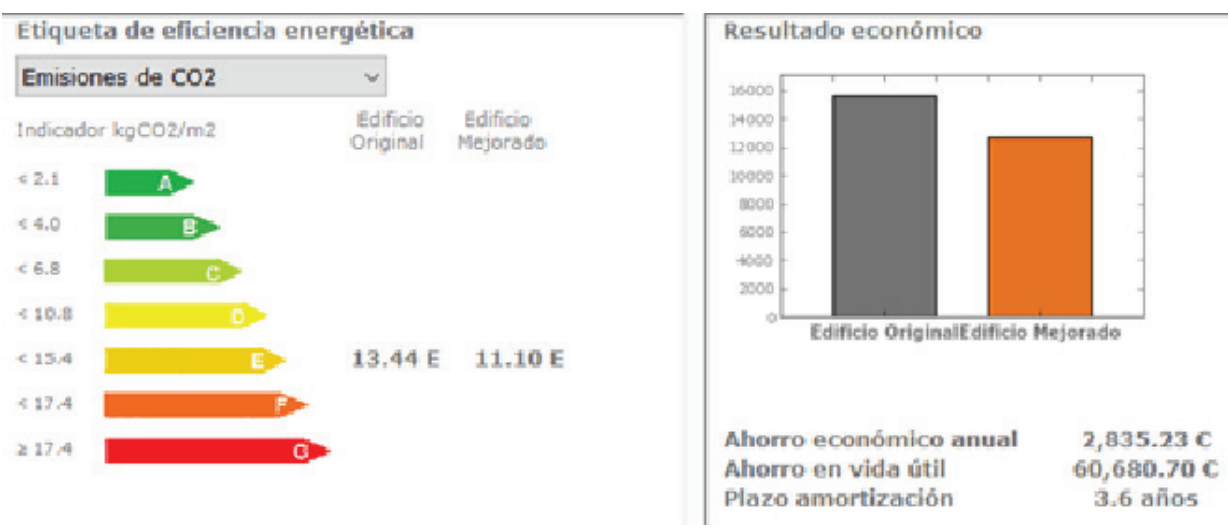

Figura 11. Viabilidad económica del conjunto de medidas propuestas desde el punto de vista estructural del edificio. Fuente: foto de pantalla del complemento Saltoki Instalaciones de CE3X.

A partir de estos análisis de viabilidad de las medidas propuestas, tanto en términos energéticos como económicos, la propuesta final fue de aplicar medidas relacionadas con aislamientos internos en los muros de la fachada suroeste, ya que representa la zona más caliente del edificio, así como mantener la medida del cambio de las ventanas, pues, aunque su rentabilidad energética no es la mejor, existe una necesidad real de mejorarlas. Por otro lado, aun cuando el uso de un sistema fotovoltaico puede ser de mucha ayuda para mejorar la certificación energética del edificio, el área útil para introducir esta tecnología es muy limitada, por lo que el proyecto aprobado para el edificio bajo estudio tiene un máximo de 69,54 kWp.

La suma de las mejoras de incorporación de sistema fotovoltaico, la colocación del aislamiento interno y de los cambios de las ventanas ofrece un ahorro potencial total del $52,0 \%$ de las emisiones totales, tal y como puede observarse en la figura 12, siendo un pequeño aporte al problema de la contaminación ambiental del planeta, mientras se logra una disminución posible del $43 \%$ la demanda de energía para la climatización, como puede observarse, lo que representa 59532 MWh que no se consumirían en el año, lo que hace que la calificación preliminar realizada cambia hacia un nivel superior, de D a $\mathrm{C}$, al disminuir el consumo anual de energía primaria no renovable a un valor de 79,63 kWh / $\mathrm{m}^{2}$.año, lo cual puede verse en la figura 13.

Calficación energética del edificio con el conjunto de medidas de mejora

\begin{tabular}{|lccc|}
\hline RESULTADOS & Medidas mejora & Caso base & Ahorro \\
Demanda de calefacción & No calificable & No calificable & - \\
Demanda de refrigeración & $9.6 \mathrm{C}$ & $16.9 \mathrm{D}$ & $43.1 \%$ \\
Emisiones de calefacción & No calificable & No calificable & - \\
Emisiones de refrigeración & $5.3 \mathrm{D}$ & $11.7 \mathrm{~F}$ & $54.4 \%$ \\
Emisiones de ACS & $0.0 \mathrm{~A}$ & $0.0 \mathrm{~A}$ & $0.0 \%$ \\
EMISIONES GLOBALES & $6.5 \mathrm{C}$ & $13.4 \mathrm{E}$ & $52.0 \%$ \\
& & & \\
\hline
\end{tabular}

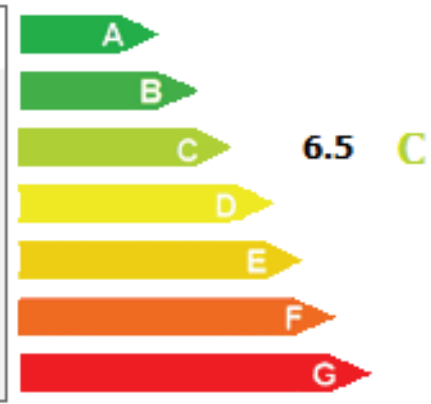

Figura 12. Certificación energética del edificio desde el punto de vista de las emisiones. Fuente: foto de pantalla del software CE3X. 


\section{Certificación anterior}

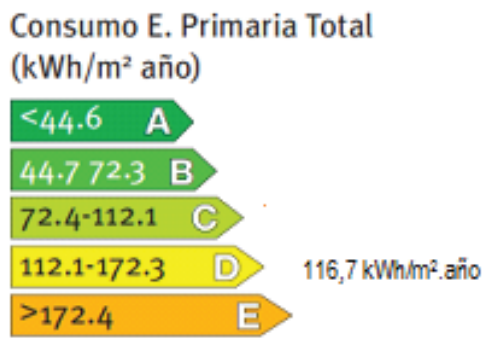

\section{Certificación con las mejoras}

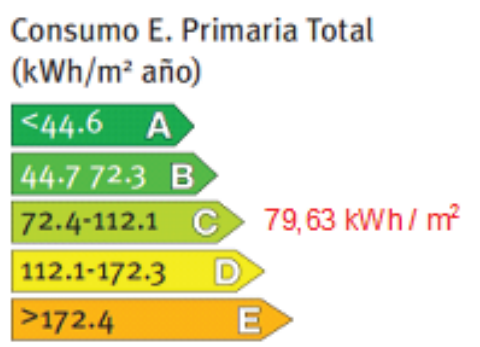

Figura 13. Comparación de la certificación energética del edificio desde el punto de vista del consumo de energía a partir de las mejoras implementadas.

\section{Conclusiones}

- La aplicación de un procedimiento para certificar energéticamente una edificación permite evaluar el estado en que se encuentra la edificación bajo estudio, así como analizar los posibles resultados de aplicar un conjunto de medidas para elevar la eficiencia energética de la instalación.

- Los resultados obtenidos, al aplicar el software de certificación CE3X, permite disminuir en un nivel, de nivel $\mathrm{D}$ a nivel $\mathrm{C}$ (en el caso del consumo de energía por área del edificio) y en dos niveles, del nivel $\mathrm{E}$ al nivel $\mathrm{C}$ (en el caso de las emisiones de $\mathrm{CO}_{2}$ por área del edificio), lo cual lleva a obtener, teóricamente, ahorros del $52 \%$ de las emisiones de $\mathrm{CO}_{2}$ y del $43 \%$ del consumo de energía asociado a la climatización.

- Las medidas propuestas para lograr los resultados anteriores se justifican económicamente por los valores del valor Actual Neto calculado (análisis de viabilidad). La inversión total necesaria para satisfacer las mejoras escogidas es 36 300,00 €, estimándose que en 8 años toda la inversión habrá sido recuperada.

\section{Referencias}

[1] "Código Técnico de la Vivienda, España", BOE-A-2006-5515, vol. 74, pp. 11816 a 11831, 2006. https://www. boe.es/eli/es/rd/2006/03/17/314

[2] D. Marqués et al. "Análisis y certificación energética de una vivienda", Consulta:25 junio, 2019. https://addi. ehu.es/handle/10810/36823

[3] M.Castro et al. "Evaluación preliminar desde el punto de vista de le eficiencia energética del edificio del CEDAI. Informe final de contrato", Documento Interno, 2019. 